\title{
13
}

\section{Development of a MPEG Data Stream Characterization for Use with ATM Networks}

\author{
Olen L. Stokes \\ IBM Corporation \\ P.O. Box 12195, Research Triangle Park, N.C., 27709 \\ (919)467-5200,olstokes@vnet.ibm.com
}

\author{
Arne A. Nilsson \\ North Carolina State University \\ Department of Electrical and Computer Engineering \\ Raleigh, N.C., 27695, (919) 515-5130, fax (919) 515-5523 \\ nilsson@eos.ncsu.edu
}

\begin{abstract}
The various parameters which affect MPEG data streams are discussed. An AR model is developed to characterize both constant bit rate and variable bit rate videos. Three videos with different bit rate modes, audio content, frame type mixture, and group of pictures sizes are analyzed. The accuracy of the AR model in predicting the size of the next frame based on previous frames is examined. Possible enhancements to the AR model are also introduced. The role of the AR model in the development of ATM networks is discussed.
\end{abstract}

ATM networks, MPEG, AR model

Keywords

\section{INTRODUCTION}

"We are moving into an age of Information Networking, with users anticipating increasing freedom to communicate with people and retrieve information anytime, anywhere, and in multiple media" (Albanese, 1991). These expectations translate into expanding markets and profits when desirable new services can be provided economically. The key to these services and their revenue growth is video. In fact, "digital video is likely to become the dominant B-ISDN 
bandwidth driver, particularly for on-demand entertainment services" (Terry, 1992). Providing this information will require higher speed services with more flexibility than is possible with today's networks. Broadband ISDN (B-ISDN) networks with Asynchronous Transfer Mode (ATM) services have been proposed to provide the needed bandwidth and versatility.

"The study of the statistical properties of packet video streams, to model video sources, is a required step in the process of designing B-ISDN networks to handle heterogeneous traffic" (Pancha, 1994). For network control, the ability to predict data rates could enable effective congestion control measures. For a constant bit rate (CBR) connection, the amount of bandwidth required and the degree of multiplexing possible are easily determined at connection setup. The task becomes to insure that this guaranteed traffic arrives with an acceptable delay and jitter. However, for a variable bit rate (VBR) video connection, the required bandwidth and resulting multiplexing must be adjusted over time. By predicting the upcoming requirements, the network can adjust its allocations to meet the new conditions. Likewise, an encoder could request a new allocation based on its predictions (Pancha, 1993).

\section{MPEG VIDEO}

In order to effectively utilize the available bandwidth and to provide the desired picture quality, compressed video encoding/decoding methods are being developed. One such standard which is receiving much attention has been developed by the Moving Pictures Expert Group (MPEG). The current standard is commonly known as MPEG-1 and is directed at providing image quality comparable to VHS video and sound quality similar to audio CDs. Although it is targeted for digital storage media, MPEG "is flexible enough to be used in a variety of video applications" (Pancha, 1993) including transmission through B-ISDN ATM networks. Also, an MPEG-2 standard is being developed for higher resolution images and correspondingly higher data rates.

The data stream from an MPEG video is inherently variable. This comes in part from the three different algorithms for encoding a video picture. An intra-coded picture, or I-frame, is an encoding of the picture based entirely on the information in that frame. A predictive-coded picture, or P-frame, is based on motion compensated prediction between that frame and the previous reference frame (I- or P-frame). A bidirectionally predictive-coded picture, or B-frame, is based on motion compensated prediction between that frame and the previous or next reference frame (I- or P-frame). The size of the resulting frame varies significantly between frame types. I-frames are the largest while B-frames are the smallest.

Further, within each frame type, the size of the resulting encoded information varies. The size of an I-frame varies based on picture content. P- and B-frames vary depending on the motion present in the scene as well as picture content. Also, the quantizer-scale parameter $(q)$ can be varied by the encoder to change the size and corresponding quality of each frame. A small $q$ value produces a higher quality picture that requires a larger data rate. A large $q$ value generates a smaller data rate at the expense of a lower quality picture. The value(s) chosen by the encoder are determined by the desire to either provide a constant picture quality or maintain a particular data bit rate.

An MPEG data stream which contains audio as well as image data also includes system level control information. The control information defines the mixing of data packets which contain either audio or image data. This interleaving of audio and video packets is not necessarily 
constant, nor are the audio packets always equally sized. Therefore, the audio data rate also varies over certain time scales.

An MPEG encoder chooses between two basic service types: constant bit rate (CBR) and variable bit rate (VBR). Note that bit rate refers to the rate at which data is written to or taken from storage media (such as a CD-ROM) or transmitted over a network. For VBR video, the data rate is allowed to vary over time while the picture quality potentially remains constant. For CBR video, the picture quality is varied to insure that the resulting data stream can be written, read, or transmitted at a constant rate. To accomplish this, the data rate and picture quality are manipulated during encoding by adjusting $q$.

This CBR data stream does not imply a constant frame size. Rather, it means that the output of an encoder buffer can be read at a constant bit rate. By using a sufficiently large buffer which is partially filled prior to removing the first video information, and by varying $q$ so that the buffer never overruns or underruns, the encoder can produce a data stream which appears to be a CBR source to the remainder of the system. The encoder system shown in Figure 1 can operate in such a CBR manner or in a VBR manner. The CBR versus VBR decision is made at encoding time.

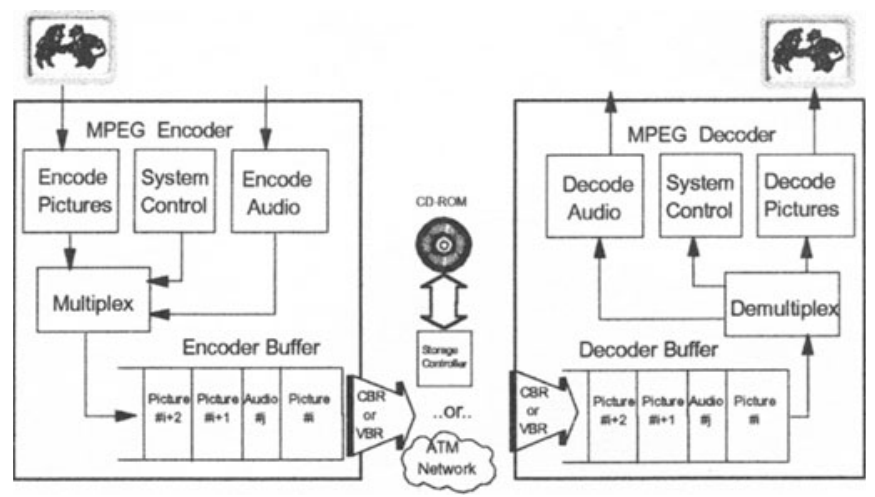

Figure 1 MPEG encoding and decoding systems.

The MPEG data shown in Figure 1 is part of a system level data stream as discussed above. The control information is not shown, nor are any padding data packets which might be required to obtain a CBR stream. The system and pad data rates are significantly smaller than the video and audio data rates. Note that many of the MPEG data streams available for analysis contain only image or audio information, but not both.

The decoder system shown in Figure 1 can also operate under CBR or VBR conditions. The decode buffer is initially filled to a level specified by the data stream before any actual picture and/or audio information is removed from the buffer and decoded. Thereafter, the buffer is filled on one end by the arriving data and emptied periodically on the other by the decoders. As long as the buffer does not overrun or underrun, the video can be displayed properly. A generic MPEG decoder, the system target decoder (STD), is defined in the MPEG standard (ISO, 1993). The encoder must insure that the data stream it produces can be played properly by a directly connected STD. Therefore, the encoder must define the decoder minimum buffer size, initial 
delay, and audio and video data rates. Note that any delay jitter introduced by transmission over a network is not necessarily anticipated by the encoder.

The initial delay to allow the decode buffer to partially fill normally causes no concern when playback is from a digital storage medium. However, when the playback is part of an interactive or real-time display, this delay may create problems. For example, an initial delay in the decode buffer of $250 \mathrm{msec}$ will significantly increase the probability that the round-trip delay will have a noticeable affect on a conversation. In these cases, a VBR mode of operation, where a frame's data is transmitted as soon as it is encoded, may be required. The encoder can either maintain a constant picture quality or continue to use the CBR algorithm without buffering. This latter mode would allow the data at the remote site to be both displayed as well as buffered for storage and future CBR playback.

The frame type (I, P, or B) selected for a particular frame is based on a repeating sequence of frames called a Group Of Pictures (GOP). Two typical groups are shown in Figure 2. The sequence used is chosen by the encoder. The MPEG standard does not completely specify the encoding process, but rather defines a syntax from which decode by the STD is possible. This allows numerous encoding options which increases the difficulties in handling and controlling MPEG traffic.

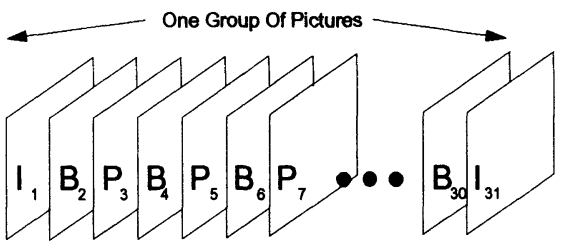

A. One l-Frame each 30 frames

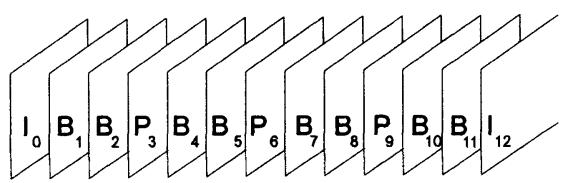

B. One I-Frame each 12 frames

Figure 2 MPEG frame display sequence.

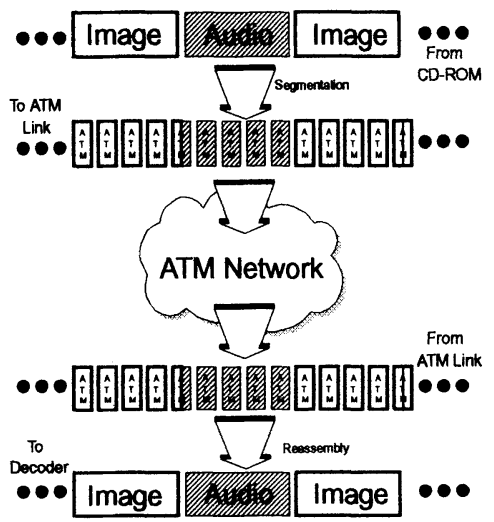

Figure 3 MPEG-1 data stream to/from ATM cells.

Once the data stream is encoded, it can be used locally or transmitted through a network. It can be displayed immediately or written to a digital storage medium for future display. Whenever the data stream is transmitted through an ATM network, the data must be divided into blocks which match the size of the ATM cell payload. This means 44- to 48-octet blocks depending on the ATM Adaptation Layer (AAL) used. At the receiving end, the data from the cell payloads is reassembled into the original MPEG stream. This process is shown in Figure 3. Should the data not be interleaved, different data types would travel on separate ATM virtual connections. 


\section{MPEG CHARACTERIZATION}

"In spite of all efforts, so far no general consensus has been reached for how to model data rates generated by typical video codecs..." (Heeke, 1993). The derivation of an MPEG-1 mathematical characterization is challenging. As discussed above, MPEG-1 data streams are affected by numerous factors including picture content, object motion, audio content, and encoder options. The result is a random sequence of frame sizes which is difficult to analyze. Figure 4 shows three examples of MPEG-1 data stream frame sizes. The sizes are in terms of ATM cells with 44-octet payloads.
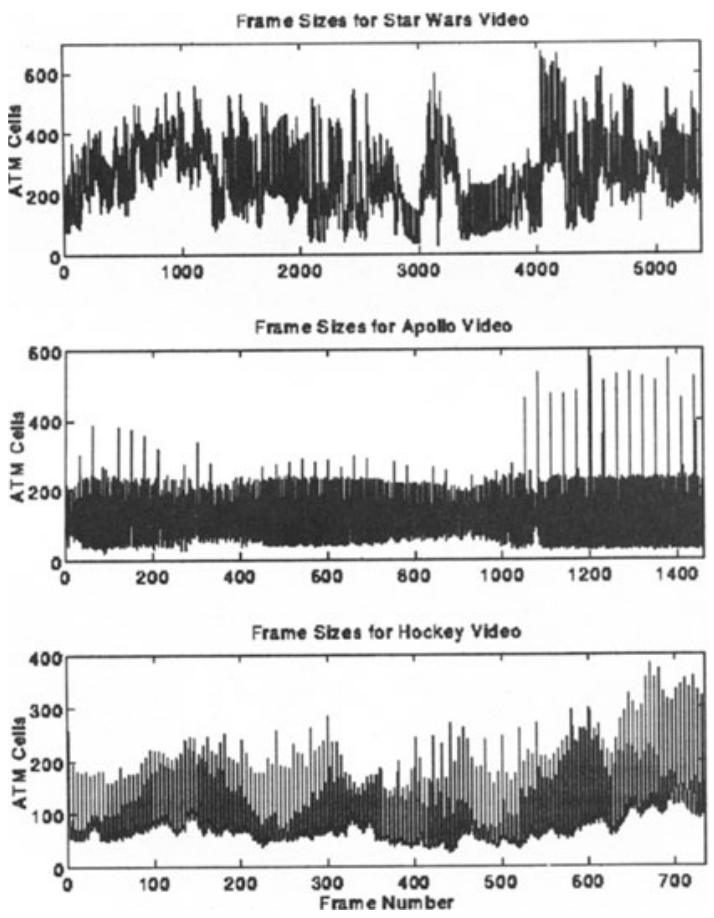

Figure 4 Frame sizes for sample video sequences.

These MPEG-1 data streams represent three different sets of encoder parameters. The Star Wars sequence is based on the image-only VBR data stream described in (Pancha, 1993). It contains only I- and P-frames and utilizes a constant $q$. The Apollo sequence is a system level CBR stream with interleaved audio and image (Aris). It includes all three frame types. The Hockey sequence is also a VBR image-only stream (North Valley Research). However, this encoding varies $q$ by frame type and uses B-frames as well as I- and P-frames. All of these videos contain camera shot changes as well as changes in movement and camera panning.

Using these frame sizes as a sequence of random numbers, the average and standard deviation can be calculated as well as the autocorrelation functions (ACFs). These first two statistics are 
shown in Table 1. The ACFs from the Hockey sequence, normalized with respect to the variance of the frame sizes, is shown in Figure 5.

Table 1 Example video sequence statistics

\begin{tabular}{ccccc}
\hline Video & $\begin{array}{c}\text { Mean } \\
(\text { ATM cells })\end{array}$ & $\begin{array}{c}\text { Standard Deviation } \\
(\text { ATM cells })\end{array}$ & $\begin{array}{c}\text { Picture } \\
\text { Sequence }\end{array}$ & $\begin{array}{c}\text { GOP Size } \\
\text { (Pictures) }\end{array}$ \\
\hline Star Wars & 227.69 & 106.09 & IPPPPPPP... & 16 \\
Apollo & 131.99 & 86.79 & IBPBPBP... & 30 \\
Hockey & 100.71 & 69.49 & IBBBBPB... & 20 \\
\hline
\end{tabular}

The properties of the GOP size and frame mixture are present in the normalized ACFs. Each of the ACFs shows a periodic peak corresponding to a lag equal to the GOP size. At this point, a frame is always being correlated with another frame of the same frame type. Since the Star Wars video utilizes only P-frames between I-frames, there is a gradual decay in the correlation between the frames until the lag reaches the same point in the GOP cycle. However, in the other two videos, there is a pattern of $\mathrm{P}$ - and $\mathrm{B}$-frames which produce additional peaks in the ACFs. These peaks also occur when similar frames are being correlated (P-frames to P-frames and Bframes to B-frames).

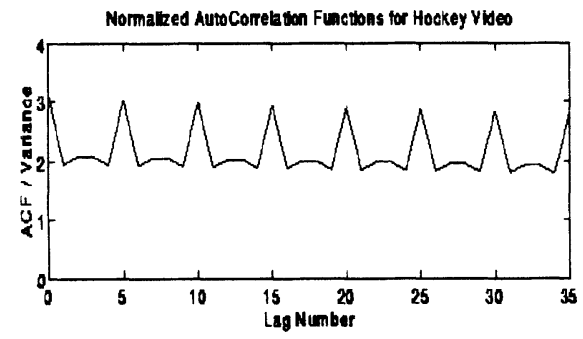

Figure 5 ACFs for Hockey video sequence.

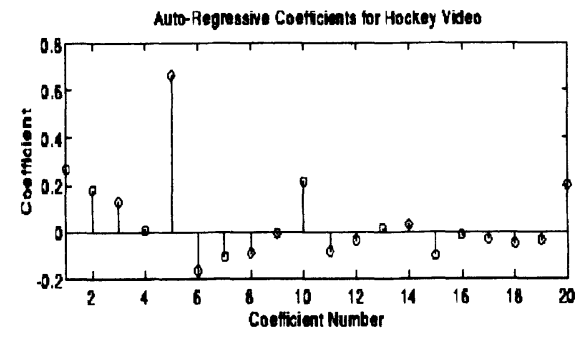

Figure 6 ARCs for Hockey video sequence.

To model the sequences, the ACFs are used to calculate autoregressive coefficients (ARCs) for each sequence (Shanmugan, 1988). The number of coefficients used is based on the GOP size, with sufficient coefficients included to allow past frames from one GOP to be utilized. The resulting Hockey ARCs are shown in Figure 6.

From the ARCs, an autoregressive (AR) model for frame sizes is created based on:

$x_{n}=\left[\sum_{i=1}^{G O P}\left(A R C_{i} x_{n-i}\right)\right]+e_{n}$

where: $x_{n}=$ frame size sequence

$A R C_{i}=$ autoregressive coefficient for lag $i$

$G O P=$ size of one group of pictures

$e_{n}=$ excitation sequence

The $e_{n}$ data stream should ideally be an uncorrelated white Gaussian noise random sequence (Shanmugan, 1988). 
To determine the characteristics of the excitation sequence, the following equation is used to extract the sequence:

$e_{n}=x_{n}-\left[\sum_{i=1}^{G O P}\left(A R C_{i} x_{n-i}\right)\right]$

Note that the frame sizes from the frames of the first GOP are used to seed the calculation. Thus, the excitation sequence begins with the excitation value for the first frame of the second GOP. The resulting excitation sequence for Star Wars is shown Figure 7. At places where scene changes occur, the excitation sequence contains larger absolute value excitations.

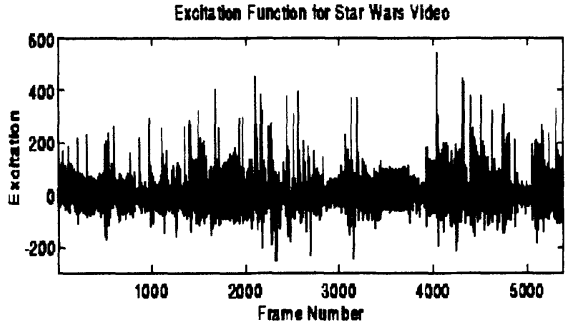

Figure 7 Excitation function for Star Wars video sequence.

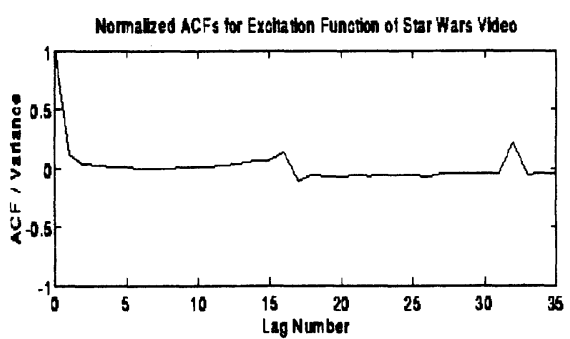

Figure 8 ACFs for Star Wars excitation function.

The ACFs for the excitation sequence are then calculated. For white-noise, the sequence should be uncorrelated (Shanmugan, 1988). As is seen in Figure 8, the derived Star Wars excitation sequence is nearly uncorrelated. There are, however, slight peaks at the values corresponding to lags which are multiples of one GOP. These peaks will be discussed in more detail below.

Further, the excitation distribution should be Gaussian with a mean of 0.0 (Shanmugan, 1988). From Table 2, all three sequences have very nearly a 0.0 mean. To allow comparison of the distributions, the excitation is normalized using the standard deviation of the excitation sequence. The resulting excitation histogram should produce a Gaussian curve with a standard deviation of 1.0. The distributions are very similar to the expected shape, as demonstrated in Figure 9. Note also that the standard deviations of the excitation sequences are considerably smaller than the standard deviations of the corresponding video sequences.

Table 2 Excitation sequence statistics

\begin{tabular}{ccc}
\hline Video & $\begin{array}{c}\text { Mean } \\
(\text { ATM cells })\end{array}$ & $\begin{array}{c}\text { Standard Deviation } \\
(\text { ATM cells })\end{array}$ \\
\hline Star Wars & 1.31 & 55.16 \\
Apollo & 0.37 & 33.59 \\
Hockey & 0.88 & 14.53 \\
\hline
\end{tabular}




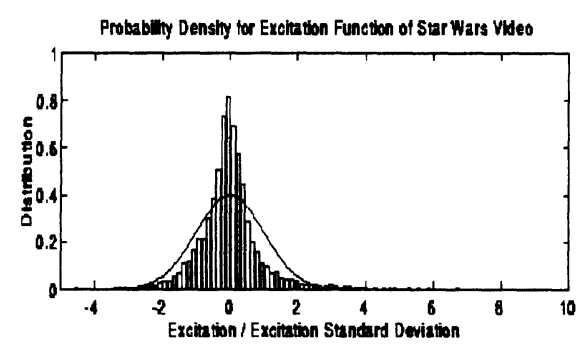

Figure 9 Excitation probability density.

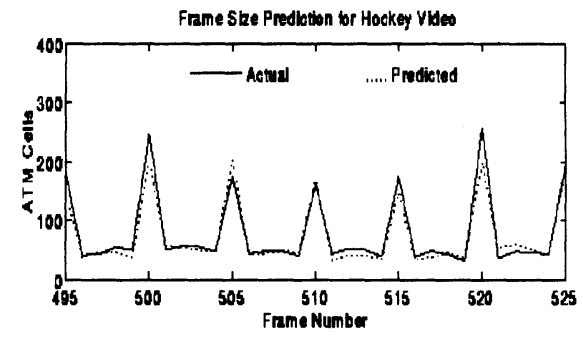

Figure 10 Prediction for Hockey video sequence.

There are, however, more excitations which exceed the three sigma $( \pm 3 \sigma)$ values than would be expected for a pure Gaussian distribution. These excitations occur at, and soon after, video scene changes. These large values increase the excitation standard deviation. This alters the relationship of the Gaussian reference curves to the displayed distributions shown in Figures 9, 12, and 14.

For network control, these calculated ARCs can predict the next frame size through the use of following equation:

$$
p_{n}=\sum_{i=1}^{G O P}\left(A R C_{i} x_{n-i}\right)
$$

where: $p_{n}=$ predicted MPEG frame sizes

The prediction begins for the first frame in the second GOP to allow seeding with actual past values. The prediction error is then be defined as:

$$
E_{n}=x_{n}-p_{n}=x_{n}-\sum_{i=1}^{G O P}\left(A R C_{i} x_{n-i}\right)
$$

where: $E_{n}=$ prediction error

A comparison of Equations 2 and 4 reveals that the resulting error sequence $E_{n}$ is identical to the excitation sequence $e_{n}$. Therefore, if the ARCs are known, the next frame size should be predictable to the accuracy demonstrated by the excitation sequence.

Unfortunately, the calculations of the ACFs and ARCs above involve the use of future knowledge. That is, the ACFs and ARCs are calculated using the entire frame size sequence. Then, those results are applied to the sequence as post-processing. This is not possible in a network control or a real-time encoding environment. In these cases, only previously received frame sizes are available. However, it should be possible to provide the size of the GOP to the network at connection setup time. Note that the mixture of frames within the GOP is not required.

To predict based only on prior frames, the ACFs are estimated based on the frame sizes that have already been received. The ARCs are then obtained from the estimated ACFs. The ACFs are calculated using the biased estimator: 
$R(m)=\frac{1}{N} \sum_{n=0}^{N-/ m /-1}\left(x_{n} x_{n+m}\right)$

where: $R(m)=$ autocorrelation function (ACF) for a lag of $m$

$N=$ Number of frame sizes included in the calculation

If calculated in this manner, the storage of all previously received frame sizes would be required. To limit the storage requirements to the number of frames in one GOP, the following calculations are made beginning with the first frame of the second GOP:

$$
\begin{aligned}
& \operatorname{Sum}_{n}(m)=\operatorname{Sum}_{n-1}(m)+\left(x_{n} x_{n-m}\right) \\
& R_{n}(m)=\frac{\operatorname{Sum}_{n}(m)}{n}
\end{aligned}
$$

where: Sum $_{0}=0.0$

$x_{-}(G O P-1) \ldots x_{0}=$ seeded values from first GOP frame sizes

$R_{n}(m)=$ estimate $n$ of ACF for lag $m$

The estimated ARCs are then calculated based on the estimated ACFs and used to predict the $n+1$ frame size using Equation 3 . Note that numerous frames must be received before the ACFs and ARCs converge to values near those shown in Figures 5 and 6. Following that, if desired to reduce calculations, the ACFs and ARCs need only to be updated periodically. However, Sum $n$ must be updated every frame.

Since the ACFs calculated in Equation 7 are not the same as those used to extract the excitation sequence $e_{n}$, the prediction error $E_{n}$ will differ from the excitation $e_{n}$. Figure 10 shows the actual and predicted frame sizes for the Hockey sequence using the above methodology. Figure 11 shows the resulting prediction error for the Apollo sequence. The ACFs and ARCs are allowed to settle for 250 frames before "steady-state" prediction is assumed. Figure 12 shows the normalized distribution of the prediction errors for the Star Wars sequence. This distribution closely resembles the excitation distribution indicating that the next frame size can be predicted within the accuracy of the excitation sequence.

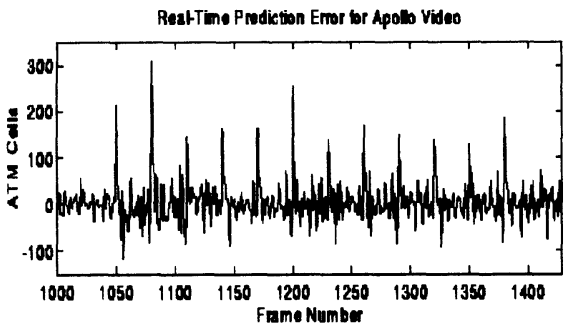

Figure 11 Prediction error for Apollo video

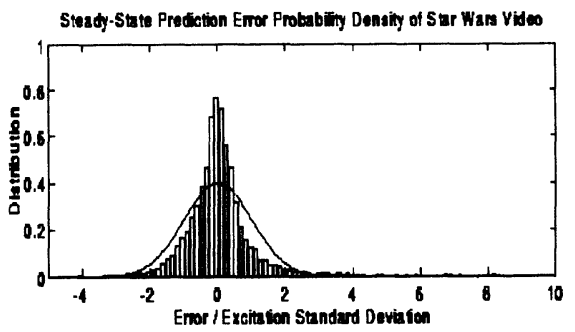

Figure 12 Prediction error probability density. sequence.

As noted above, the ACFs for the excitation sequences indicate a small correlation at lags equal to multiples of the size of the GOP. If this correlation could be used to estimate the above frame size prediction error, the uncertainty in the next frame could potentially be reduced. The 
resulting prediction algorithm would be similar to an autoregressive moving average (ARMA) calculation. The following formula is used:

$C E_{n}=E_{n}-p E_{n}=\left[x_{n}-\sum_{i=1}^{G O P}\left(A R C_{i} x_{n-i}\right)\right]-\sum_{j=1}^{G O P}\left(\operatorname{EARC}_{j} E_{n-j}\right)$

where: $C E_{n}=$ corrected prediction error

$E_{n}=$ non-corrected prediction error

$p E_{n}=$ estimated non-corrected prediction error

$E A R C=\mathrm{ARCs}$ calculated for the non-corrected prediction error $E_{n}$

A method similar to that used above for calculating the estimated ACFs and ARCs is used to estimate the coefficients (EACF and EARC) of the prediction errors shown in Figures 10-12. Once again, the coefficients must be allowed to approach "steady-state" values before the results are stable. For this estimate, 200 prediction errors are utilized. The resulting correction coefficients EARC are substantially smaller than the prediction coefficients ARC.

The resulting corrected prediction errors $C E_{n}$ are shown in Figure 13 for the Apollo sequence. The large periodic errors are noticeably reduced. The errors are again normalized by the excitation standard deviation and the Star Wars distribution is shown in Figure 14. The corrected error distribution shows marginal improvement over the non-corrected prediction errors in Figure 12 . The corrected distributions have slightly smaller standard deviations and fewer errors greater than $+4 \sigma$. This only slight improvement is reasonable considering the small size of the correlation peaks of the excitation sequence ACFs. The small EARCs also reinforce this.

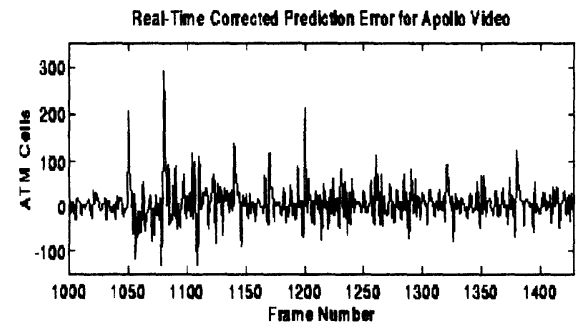

Figure 13 Corrected prediction error for Apollo video sequence.

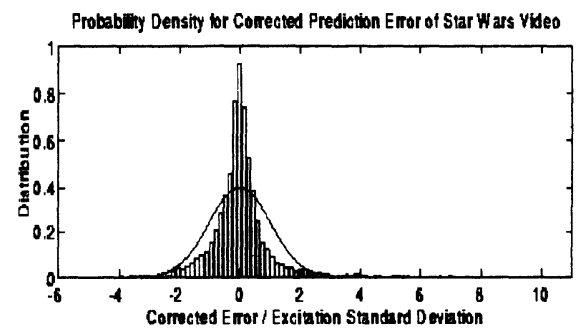

Figure 14 Corrected prediction error probability density.

\section{MPEG IN ATM NETWORKS}

The understanding obtained in the development of the above characterization of MPEG-1 video data streams can aid in the development of B-ISDN ATM networks which must transport these videos. For network control of a VBR connection, the network must manage a new load during each frame period. The more accurately that this load can be predicted, the better that statistical multiplexing can be performed. Also, a network policing function could use this predicted load to set the permitted data rate for the next frame. Should the encoder also use the same algorithm, it would know the load expected by the network for the upcoming frame. If a larger than 
expected frame is produced (corresponding to a large positive prediction error), a layered encoder similar to that discussed in (Pancha, 1993) and (Pancha, 1994) could mark an appropriate number of cells as low priority.

If the network does not provide this level of control, the encoder can also take preventive action. If the network supports a fast-reservation protocol, the encoder can request a different bandwidth allocation based on its own prediction of the next frame size. This mode of operation is well described in (Pancha, 1993) and the AR prediction method presented above provides an alternate method of predicting the next frame size.

The decision to incorporate the additional complexity of generating predicted error corrections must be made after ATM networks are better understood. While the improvement is slight, this improvement might be worthwhile in network control environments. If heavily loaded networks are found to become unstable with small increases in load, the enhanced prediction may prevent problems. Since large positive prediction error values represent large underestimates of the frame size, reduction of errors greater than $+4 \sigma$ might significantly increase stability.

Further analysis might reveal other, less complex methods which can address the repeatability (correlation) of prediction errors. A simple strategy might be to increase the predicted frame size by some percentage of the prediction error which occurred one GOP prior. This approach could even be modified to correct the prediction only when the prior error was greater than some minimum value. Further analysis is required.

\section{CONCLUSIONS}

The task of defining a perfect model of an MPEG-1 data stream remains a challenge. However, an AR model can be used to predict the next MPEG-1 frame size within the tolerances of an excitation sequence whose properties can be determined. The AR model is constructed based on the previous frame sizes and the number of frames in a GOP. This prediction can be slightly improved by correcting the estimate through the use of another AR prediction based on past errors. The resulting AR models can be used by network control functions as well as MPEG-1 encoders.

\section{REFERENCES}

Albanese, A., Bussey, H., Weinstein, S. and Wolff, R. (1991) "A Multi-Network Research Testbed for Multimedia Communications Services," IEEE International Conference on Communications, Volume 1, 3.3.1-6.

Aris Multimedia Entertainment, Inc., video sequence statistics derived from aplllnc2.mpg video on Worldview CD-ROM.

Heeke, H. (1993) "A Traffic-Control Algorithm for ATM Networks," IEEE Transactions on Circuits and Systems for Video Technology, Volume 3, Number 3, 182-9.

ISO Committee 11172 (1993) "Coding of moving pictures and associated audio for digital storage media at up to $1.5 \mathrm{Mbit} / \mathrm{s}$," ISO/IEC JTC 1/SC 29. 
North Valley Research, video sequence statistics derived from hockeyl.sif.variable video.

Pancha, P. and El Zarki, M. (1993) "Bandwidth-Allocation Schemes for Variable-Bit-Rate MPEG Sources in ATM Networks," IEEE Transactions on Circuits and Systems for Video Technology, Volume 3, Number 3, 190-8.

Pancha, P. and El Zarki, M. (1994) "MPEG Coding For Variable Bit Rate Transmission," IEEE Communications, Volume 32, Number 5, 54-66.

Shanmugan, K. S. and Breipohl, A. M. (1988) Random Signals - Detection, Estimation, and Data Analysis, Wiley, 250, 316, and 599.

Terry, J. (1992) "Alternative Technologies and Delivery Systems for Broadband ISDN Access," IEEE Communications Magazine, Volume 30, Number 8, 58-64,.

\section{BIOGRAPHIES}

Olen L. Stokes received the B.S., M.S., and $\mathrm{PhD}$. degrees from North Carolina State University in 1974, 1981, and 1995, respectively. He joined the IBM Corporation in 1974 working on communications controllers. From 1978 to 1984, he developed both hardware and microcode for the 3687 supermarket scanner, which was the first industrial user of holography. From 1985 to 1989 he was a manager in IBM's Systems Network Architecture group. He then began working in the development of high speed communications products. He is currently a Senior Engineer in the IBM Networking Hardware Division in Research Triangle Park, N.C.

Arne A. Nilsson received the M.S. and Ph.D. degrees from Lund Institute of Technology, Lund, Sweden in 1968 and 1976, respectively. He is currently a Professor with the Department of Electrical and Computer Engineering at North Carolina State University, Raleigh, North Carolina. In 1988 he became Director of the Center for Advanced Computing and Communications (formerly the Center for Communications and Signal Processing) and is now the Technical Director of that Center. He has published more than 70 papers in technical journals and conferences and has consulted for many government agencies and corporations. 